\title{
Long non-coding antisense RNA controls Uchl1 translation through an embedded SINEB2 repeat
}

\author{
Claudia Carrieri ${ }^{1 *}$, Laura Cimatti ${ }^{1 *}$, Marta Biagioli ${ }^{1,2}$, Anne Beugnet ${ }^{3}$, Silvia Zucchelli ${ }^{1,2}$, Stefania Fedele ${ }^{1}$, Elisa Pesce ${ }^{3}$, \\ Isidre Ferrer ${ }^{4}$, Licio Collavin ${ }^{5,6}$, Claudio Santoro ${ }^{7}$, Alistair R. R. Forrest ${ }^{8}$, Piero Carninci ${ }^{8}$, Stefano Biffo ${ }^{3,9}$, Elia Stupka ${ }^{10}$ \\ \& Stefano Gustincich ${ }^{1,2}$
}

Most of the mammalian genome is transcribed ${ }^{1-3}$. This generates a vast repertoire of transcripts that includes protein-coding messenger RNAs, long non-coding RNAs (lncRNAs) and repetitive sequences, such as SINEs (short interspersed nuclear elements). A large percentage of ncRNAs are nuclear-enriched with unknown function ${ }^{4}$. Antisense lncRNAs may form sense-antisense pairs by pairing with a protein-coding gene on the opposite strand to regulate epigenetic silencing, transcription and mRNA stability ${ }^{5-10}$. Here we identify a nuclear-enriched lncRNA antisense to mouse ubiquitin carboxyterminal hydrolase L1 (Uchl1), a gene involved in brain function and neurodegenerative diseases ${ }^{11}$. Antisense Uchl1 increases UCHL1 protein synthesis at a post-transcriptional level, hereby identifying a new functional class of lncRNAs. Antisense Uchl1 activity depends on the presence of a $5^{\prime}$ overlapping sequence and an embedded inverted SINEB2 element. These features are shared by other natural antisense transcripts and can confer regulatory activity to an artificial antisense to green fluorescent protein. Antisense Uchll function is under the control of stress signalling pathways, as mTORC1 inhibition by rapamycin causes an increase in UCHL1 protein that is associated to the shuttling of antisense Uchl1 RNA from the nucleus to the cytoplasm. Antisense Uchll RNA is then required for the association of the overlapping sense protein-coding $\mathrm{mRNA}$ to active polysomes for translation. These data reveal another layer of gene expression control at the post-transcriptional level.

To discover non-coding antisense transcripts of sense-antisense (S-AS) pairs expressed in the brain, the mouse syntenic loci of genes involved in neurodegenerative diseases were identified computationally and examined in the Ensembl browser (http://www.ensembl.org). The FANTOM2 clone 6430596G22 was classified as a spliced antisense lncRNA of the Uchl1 gene ${ }^{11}$; we refer to this as antisense Uchl1. UCHL1 is a neuron-restricted protein that acts as a deubiquitinating enzyme, ubiquitin ligase or monoubiquitin stabilizer ${ }^{12}$. An in-frame deletion in the Uchll gene, as in gracile axonal dystrophy mice, leads to ataxia and axonal degeneration. Although an association of UCHL1 gene mutations to familial Parkinson's disease has not been confirmed in independent families, oxidative inactivation of UCHL1 protein has been reported in Parkinson's disease and Alzheimer's disease brains ${ }^{13-15}$.

Antisense Uchl1 is a $5^{\prime}$ head-to-head transcript that initiates within the second intron of $U c h l 1$ and overlaps the first 73 nucleotides of the sense mRNA including the AUG codon. By 5' rapid amplification of cDNA ends (RACE), the transcriptional start site (TSS) of antisense Uchl1 was mapped to the second intron of Uchl1 (Fig. 1a). The nonoverlapping part of the transcript contains two embedded repetitive sequences, SINEB1 of the F1 subclass (Alu) and SINEB2 of the B3 subclass, identified by Repeatmasker ${ }^{16-18}$. The FANTOM2 clone spans a genomic region of 70 kilobases (kb) (Fig. la) and its genomic organization is conserved in mammals (Supplementary Fig. 1a).

Sense and antisense Uchl1 expression in mouse and human tissues was similar (Fig. 1b and Supplementary Fig. 1b). In the mouse, antisense Uchl1 RNA was highly expressed in the ventral midbrain (Fig. 1b) and in the MN9D dopaminergic cell line (data not shown). Mature Uchl1 mRNA was observed mainly in the cytoplasm of dopaminergic neurons, whereas antisense Uchll was enriched in the nucleus (Fig. 1c and Supplementary Fig. 2). Antisense Uchl1 expression was confirmed by qRT-PCR from dopaminergic neurons purified with laser capture microdissection (LCM, Supplementary Fig. 3).
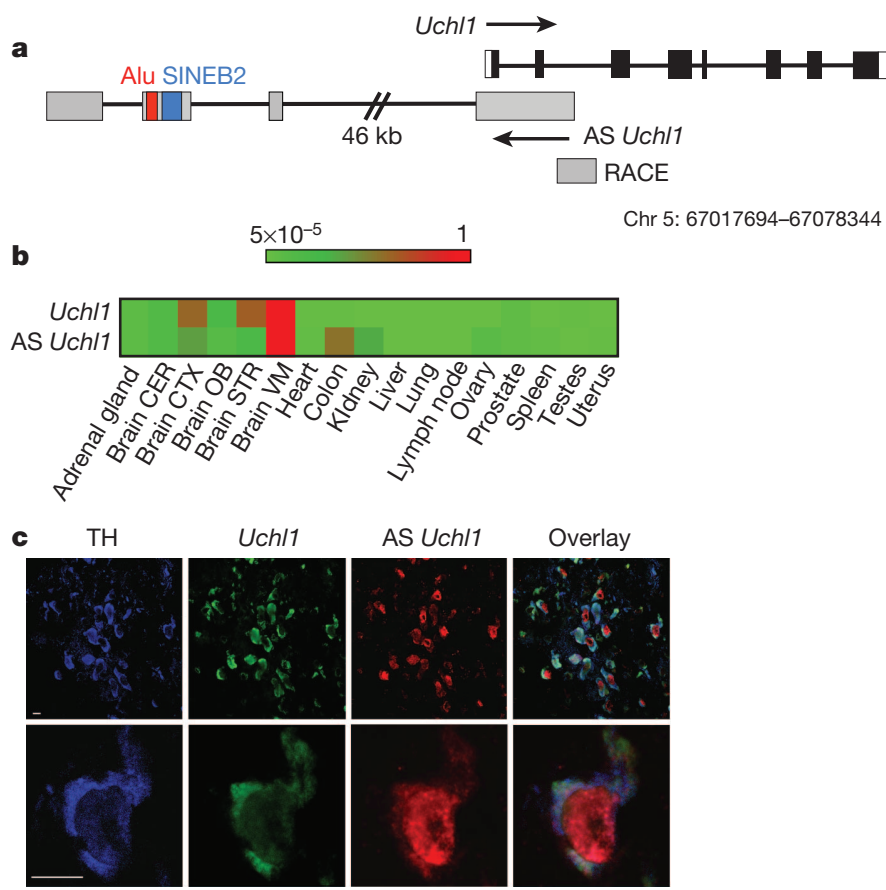

Figure 1 | Expression of antisense $\boldsymbol{U}$ chl1 in dopaminergic neurons. a, $U$ chl1/ antisense (AS) Uchl1 genomic organization. Uchl1 exons are in black; $3^{\prime}$ and $5^{\prime}$ UTRs are in white; antisense Uchll exons are grey; repetitive elements are in red (Alu) and blue (SINEB2). Introns are indicated as lines. b, Quantitative expression of $U c h l 1$ and antisense $U c h l 1$ in mouse tissues $\left(\Delta \Delta \mathrm{Ct} / \Delta \Delta \mathrm{Ct} \mathrm{t}_{\max }\right)$. CER, cerebellum; CTX, cortex; OB, olfactory bulb; STR, striatum; VM, ventral midbrain. c, Antisense Uchll (red) and Uchl1 (green) transcripts are expressed in the nucleus and cytoplasm of TH-positive dopaminergic neurons of the substantia nigra (blue).

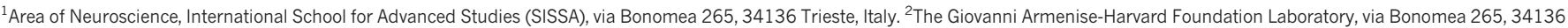

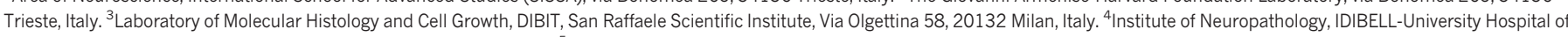

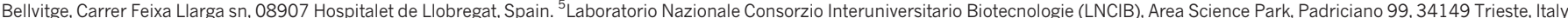

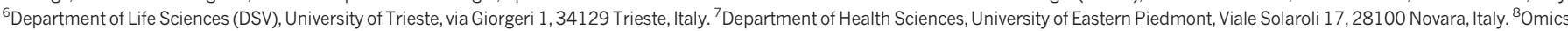

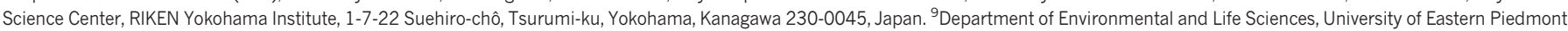
Viale T. Michel 11, 15121 Alessandria, Italy. ${ }^{10}$ Center for Translational Genomics and Bioinformatics, San Raffaele Scientific Institute, Via Olgettina 58,20132 Milan, Italy.

*These authors contributed equally to this work. 
Transient expression of antisense Uchl1 in MN9D cells caused no significant change in endogenous Uchl1 mRNA expression. Notably, a strong and reproducible upregulation of UCHL1 protein was detected within $24 \mathrm{~h}$ (Fig. 2a). When increasing amounts of antisense $U$ chl1 were co-transfected with murine Uchll into HEK cells, which do not express either transcript, dose-dependent UCHL1 protein upregulation was recorded in the absence of any significant change in the quantity of exogenous Uchl1 mRNA (Fig. 2b). These data indicate that antisense Uchl1 regulates UCHL1 expression at a post-transcriptional level.

Antisense $U c h l 1$ deletion constructs lacking the $5^{\prime}$ first exon (antisense $\operatorname{Uchl1}\left(\Delta 5^{\prime}\right)$ ) or the last three exons (antisense $\operatorname{Uchl1}\left(\Delta 3^{\prime}\right)$ ) failed to

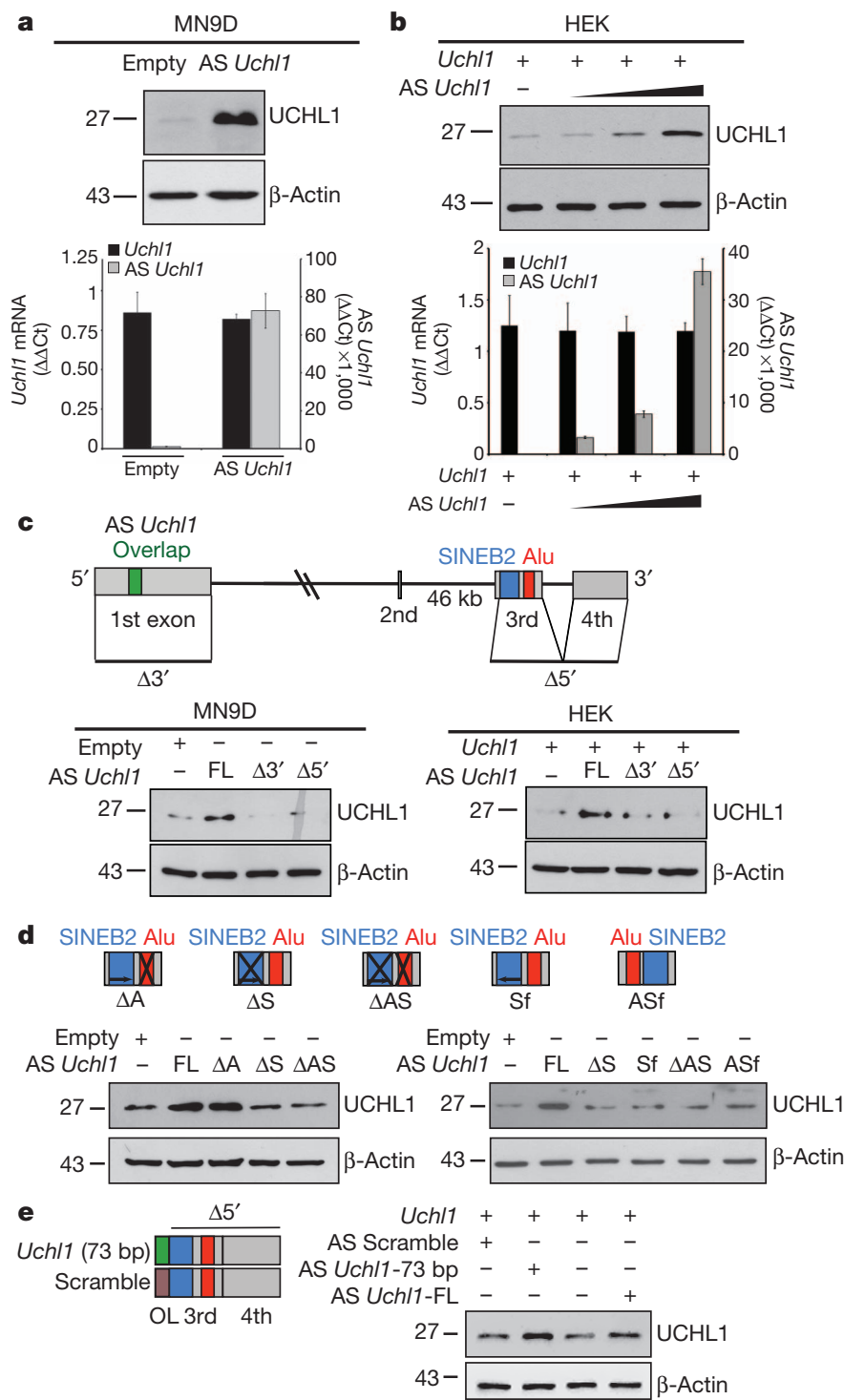

Figure $2 \mid$ Antisense Uchl1 regulates UCHL1 protein levels via an embedded inverted SINEB2 element. a, Antisense Uchll-transfected dopaminergic MN9D cells show increased levels of endogenous UCHL1 protein, with unchanged mRNA quantity. b. Increasing doses of transfected antisense Uchll titrate UCHL1 protein but not mRNA levels in HEK cells. Data in a and b indicate mean \pm s.d., $n \geq 3$. c, Full-length (FL) antisense Uchll is required for regulating endogenous (MN9D cells, left panel) and overexpressed (HEK cells, right panel) UCHL1 protein levels. Scheme of $\Delta 5^{\prime}$ or $\Delta 3^{\prime}$ deletion mutants is shown. $\mathbf{d}$, Inverted SINEB2 is sufficient to control endogenous UCHL1 protein levels in MN9D cells. Scheme of mutants is shown in $5^{\prime}$ to $3^{\prime}$ orientation. $\triangle \mathrm{A}$, $\triangle$ Alu; $\Delta$ S, $\triangle$ SINEB2; $\triangle \mathrm{AS}, \triangle \mathrm{Alu}+$ SINEB2; Sf, SINEB2 flipped; ASf, Alu +SINEB2 flipped. e, A 73-bp overlap (OL) of antisense Uchl1 is sufficient to increase UCHL1 in transfected HEK cells. Scheme of mutant and scramble control in $5^{\prime}$ to $3^{\prime}$ orientation. Units for numbers along the left of gels in a-e indicate $\mathrm{kDa}$. induce UCHL1 protein in MN9D and HEK cells, suggesting that both $5^{\prime}$ and $3^{\prime}$ components are important to antisense Uchll function (Fig. 2c and Supplementary Fig. 4a). Targeted deletion of the region containing the embedded SINEB2 and Alu repetitive sequences $(\triangle \mathrm{AS})$ was also able to prevent UCHL1 protein induction. Deletion of each repetitive element separately revealed that SINEB2 is the functional unit required by antisense Uchl1 for increasing UCHL1 protein synthesis (Fig. 2d). In all cases no change in Uchl1 mRNA level was detected (Supplementary Fig. 4b). A mutant with a flipped SINEB2 sequence was unable to increase UCHL1 protein levels, thus proving the orientationdependent activity of the SINEB2 domain embedded within antisense Uchl1 (Fig. 2d). Importantly, an artificial construct containing the 73nucleotide overlapping sequence immediately close to the repetitive elements in antisense Uchl1 $\left(\Delta 5^{\prime}\right)$ increased UCHL1 levels as much as the full-length clone (Fig. 2e and Supplementary Fig. 4c).

We then considered whether other SINEB2-containing lncRNAs may post-transcriptionally regulate the expression of their proteincoding partner, on the basis of similar structural elements. The FANTOM3 collection of non-coding cDNAs was bioinformatically screened for natural antisense transcripts that contain SINEB2 elements of the B3 subclass in the correct orientation and 5' head-to-head overlapping to a protein-coding gene. This identified 31 S-AS pairs similar to the Uchl1/antisense Uchl1 structure (Supplementary Fig. 5). By sequence alignment, we chose antisense Uxt (4833404H03), antisense of ubiquitously expressed transcript $(U x t)$, as the one with the most similar SINEB2 elements (Fig. 3a). Transfection of antisense Uxt in MN9D cells elicited an increase of UXT protein level with no change in $U x t$ mRNA (Fig. 3b), indicating that antisense Uxt was similarly able to increase protein levels post-transcriptionally.

These data indicate a model whereby lncRNAs regulate protein synthesis through the combined activities of two domains. The $5^{\prime}$ antisense

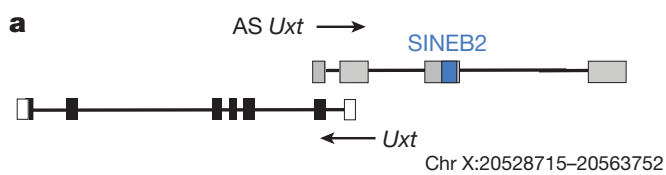

b

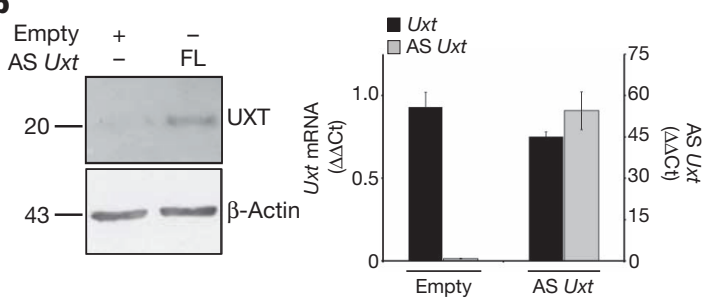

c

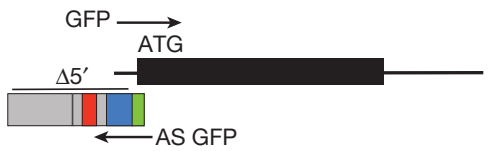

d GFP $+\quad+\quad+$

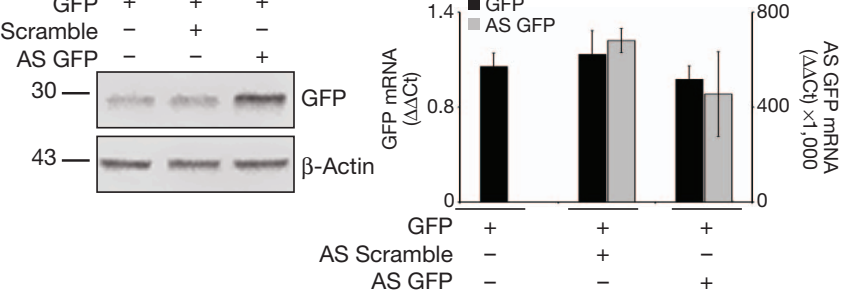

Figure $3 \mid$ Natural and synthetic antisense lncRNAs increase target protein levels. a, Scheme of Uxt/antisense Uxt genomic organization. b, Antisense Uxt increases endogenous UXT protein levels (left) without affecting RNA levels (right) in transfected MN9D cells. c, Scheme of antisense GFP construct. $\Delta 5^{\prime}$ with repetitive elements (SINEB2, blue; Alu, red) and overlap (green) regions is indicated. d, Inverted SINEB2 plus the overlap sequence increase GFP levels in transfected cells. Data in $\mathbf{b}$ and $\mathbf{d}$ indicate mean \pm s.d., $n \geq 3$. 
region provides specificity for the sense target gene whereas the repetitive element confers the protein synthesis activation domain. The model predicts that by swapping the overlapping sequence one may increase the amount of proteins encoded by the mRNAs of choice acting at the post-transcriptional level. We thus synthesized a 72-nucleotide-long artificial sequence antisense to the AUG-containing region as transcribed from pEGFP, and inserted it into antisense $U \operatorname{chll}\left(\Delta 5^{\prime}\right)$ to generate antisense GFP (Fig. 3c). Co-transfection of antisense GFP with pEGFP strongly increased GFP protein but not mRNA levels in HEK cells (Fig. 3d). When we pulsed cells with methionine for an hour and immunoprecipitated GFP, antisense GFP induced an increase in radioactively labelled, neo-synthesized GFP, without affecting mRNA levels (Supplementary Fig. 6).

To understand how the antisense Uchl1 transcript operates and the physiological conditions in which it might act, we assayed several stimuli and/or drugs for their ability to modulate UCHL1 protein expression. Inhibition of mTORC1 signalling favoured an increase in UCHL1 levels in a range from 1.5- to 2.5-fold (Fig. 4a). This effect was evident with as low as $20 \mathrm{nM}$ rapamycin (Supplementary Fig. 7) and was concomitant with dephosphorylation of mTOR targets p70S6K and 4E-BP1. Furthermore, the effect was not due to a stabilization of the protein, as co-application of cycloheximide decreased UCHL1 protein levels (Supplementary Fig. 8a). These data are surprising because rapamycin impairs formation of the CAP-dependent complex and hence translation of highly structured mRNAs ${ }^{19}$. However, in agreement with previous reports, in our experimental settings mTORC1 inhibition only slightly impairs the global rate of translation (Supplementary Fig. 8b). Under these conditions, it has been proposed that rapamycin may affect competition among different mRNAs ${ }^{20}$. If so, we proposed that inhibition of the CAP complex formation favours the translation of Uchl1 mRNA with a mechanism that requires antisense Uchl1.

To test this model, we used two complementary approaches to establish a loss-of-function phenotype. First, we downregulated antisense Uchl1 levels with short hairpin RNA (shRNA) targeting its promoter region. MN9D cells constitutively expressing shRNA for antisense Uchl1 did not show any changes in UCHL1 protein levels upon rapamycin treatment, whereas scramble control cells showed UCHL1 upregulation as in the parental line (Fig. 4b). Dephosphorylation of p70S6K and 4EBP1 proved that rapamycin inhibited mTOR activity as expected. We then exploited the dominant-negative property of an antisense Uchll mutant lacking the SINEB2 repeat element. Overexpression of antisense $U$ chl1( $\triangle$ SINEB2) inhibited the ability of full-length antisense Uchl1 to increase protein levels (Supplementary Fig. 9). Upon rapamycin treatment, cells stably expressing antisense $U$ chl1( $\triangle$ SINEB2) did not show any UCHL1 protein induction despite dephosphorylation of mTOR targets (Fig. 4c). These complementary experiments prove that functional antisense expression is required for UCHL1 protein increase elicited by rapamycin.

Because antisense Uchll transcript is enriched in the nucleus of dopaminergic neurons, we measured antisense Uchl1 and Uchll RNA content in the nucleus and cytoplasm of MN9D cells upon rapamycin treatment. As shown in Fig. 4d, rapamycin substantially increased antisense Uchl1 concentration in the cytoplasmic fraction. This effect was confirmed by a concomitant decrease in its nuclear steady-state levels, and by the absence of any de novo transcription. The total content of primary and spliced transcripts remained constant (data not shown). Uchll mRNA showed no change in subcellular distribution, de novo transcription or total cellular content. These data demonstrate that antisense $U$ chll localization can be regulated by the mTOR pathway, and its cytoplasmic level correlates with the expression of UCHL1 protein.

We therefore monitored the association of Uchl1 mRNA with polysomes to assess the role of translation in antisense Uchl1-mediated UCHL1 protein induction. Fractionated MN9D cell extracts were used to measure the recruitment of Uchl1 mRNA on polysomes by
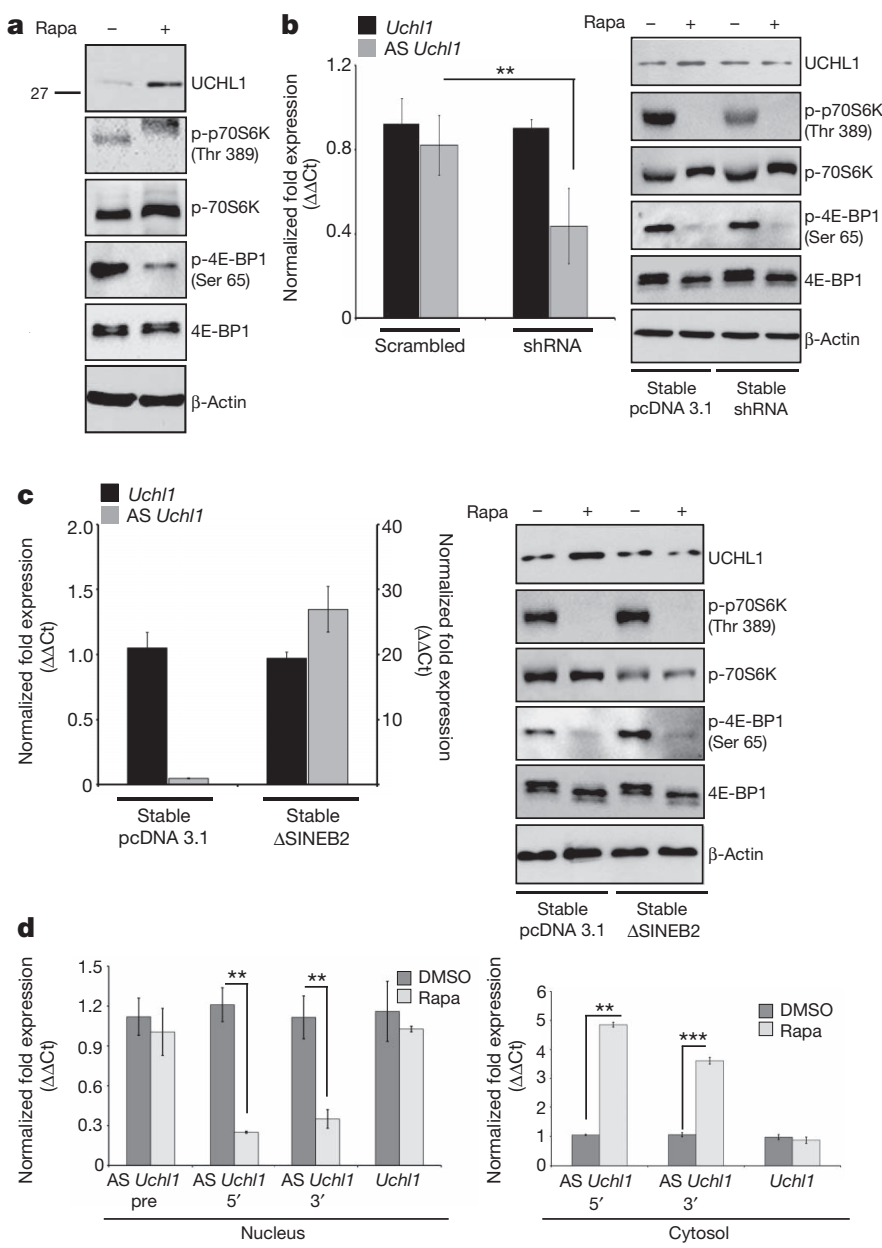

pcDNA $3.1 \quad \triangle$ SINEB2
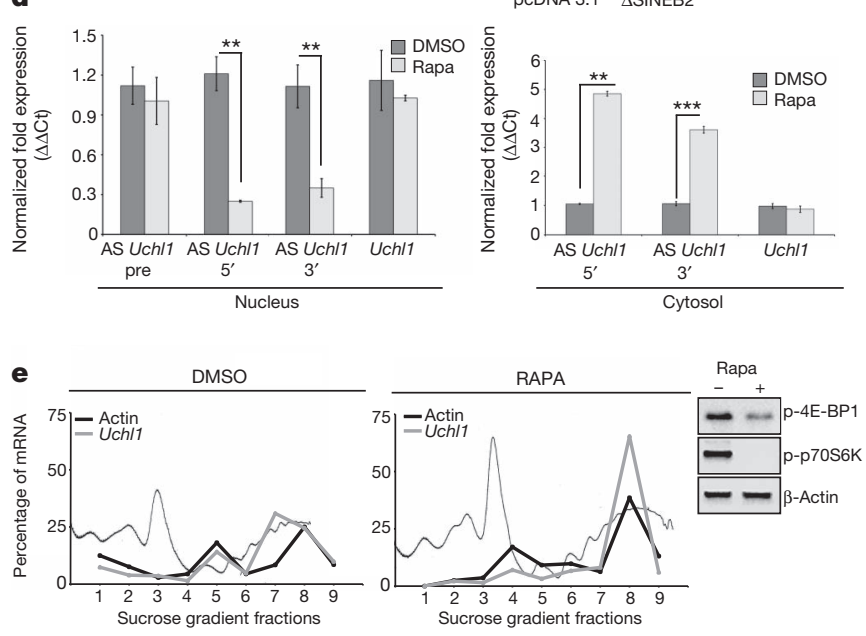

Figure $4 \mid$ Antisense Uchl1 mediates UCHL1 protein induction by rapamycin. a, UCHL1 protein level is increased in rapamycin-treated MN9D cells. Rapamycin inhibition of mTOR pathway is verified with anti-p-p70S6K and anti-p-4E-BP1 antibodies. b, Silencing antisense Uchl1 transcription (shRNA) in MN9D cells inhibits rapamycin-induced UCHL1 protein level. Left, mRNA levels; right, protein levels. c, Deletion of embedded SINEB2 ( $\triangle$ SINEB2) is sufficient to inhibit rapamycin-induced UCHL1 protein upregulation. d, Antisense $U c h l 1$ translocates to the cytoplasm upon rapamycin treatment in MN9D cells. mRNA levels were measured with $5^{\prime}$ or $3^{\prime}$ primers. Data in $\mathbf{b}-\mathbf{d}$ indicate mean \pm s.d., $n \geq 3$. $* * P<0.01 ; * * * P<0.005$.

e, Rapamycin increases Uchll mRNA in heavy polysomes; the absorbance profile is outlined in the background of each plot.

qRT-PCR and northern blotting (Fig. 4e and Supplementary Fig. 10). In basal conditions, Uchl1 mRNA was associated with translating ribosomes. Rapamycin treatment induced a shift of Uchll mRNA to heavier polysomes, consistent with an enhanced rate of translation initiation; this increase of Uchll mRNA association to heavier polysomes did not occur in cells overexpressing the dominant-negative form of antisense Uchl1 (Supplementary Fig. 11).

Antisense $U c h l 1$ is the representative member of a new functional class of IncRNAs that are part of S-AS pairs in the mammalian genome that require overlap at the $5^{\prime}$ end and the action of a SINEB2 repeat. This new function for SINEB2 sequences in the cytoplasm adds to their 
well-established role in the nucleus as inhibitors of RNA polymerase $\mathrm{II}^{16}$. Stress-dependent nucleocytoplasmic shuttling of lncRNAs may be a common strategy to regulate translation, as CTN-RNA, another nuclear-retained lncRNA, was found to have a cryptic protein-coding sequence at its $3^{\prime}$ end when in the cytoplasm ${ }^{21}$.

It is intriguing that this nuclear lncRNA-mediated mechanism for post-transcriptional control of gene expression is active when CAPdependent translation is inhibited by rapamycin. This drug blocks mTORC1 kinase, which activates the eIF4F complex ${ }^{19,22}$. However, some mRNAs escape mTORC1 inhibition by being able to be recruited to ribosomes in an eIF4F-independent manner for presenting complex mRNA loops that function as internal ribosomal entry sites (IRES) ${ }^{23}$. Indeed, IRES-mediated translation is prominent in conditions of stress or growth factor inhibition and its alteration affects processes such as tumorigenesis ${ }^{22,24}$. In genetic and neurochemical models of Parkinson's disease, mTORC1 inhibition protects dopaminergic neurons from apoptosis $^{25,26}$.

Antisense lncRNA-mediated translation may be another mechanism to maintain synthesis of pro-survival proteins, such as UCHL1, that are involved in rapamycin neuroprotective function and more generally in cellular response to stress. This mechanism may represent the outcome of an evolutionary pressure on the genomic organization of anti-stress elements to favour gene-specific regulation of translation when CAPdependent initiation is reduced. Finally, natural and synthetic antisense transcripts with embedded repetitive elements may represent molecular tools to increase translation of selected mRNAs, defining a potential new class of RNA therapeutics.

\section{METHODS SUMMARY}

5' RACE for antisense Uchl1 was performed with Gene Racer (Invitrogen). Double-fluorescence in situ hybridization of biotin- and digoxigenin-labelled probes was detected using fluorochrome-conjugated reagents. Images were captured with Confocal Laser Microscopy (LEICA). Expression of antisense Uchl1 was performed on neuronal cells lines and dopaminergic neurons purified with LCM from TH-GFP mice. Cell lines were cultured under standard conditions. shRNA targeting the promoter of antisense Uchll was cloned in pSUPERIOR.Neo.GFP vector (Oligo Engine). Polysomes were prepared by sucrose gradient and associated Uchll mRNA was measured by qRT-PCR and northern blotting.

Full Methods and any associated references are available in the online version of the paper.

Received 3 December 2010; accepted 14 August 2012.

Published online 14 October 2012.

1. Birney, E. et al. Identification and analysis of functional elements in $1 \%$ of the human genome by the ENCODE pilot project. Nature 447, 799-816 (2007).

2. The FANTOM Consortium. The transcriptional landscape of the mammalian genome. Science 309, 1559-1563 (2005).

3. Kapranov, P. Willingham, A. T. \& Gingeras, T. R. Genome-wide transcription and the implications for genomic organization. Nature Rev. Genet. 8, 413-423 (2007),

4. Kapranov, P. et al. RNA maps reveal new RNA classes and a possible function for pervasive transcription. Science 316, 1484-1488 (2007)

5. Beltran, M. et al. A natural antisense transcript regulates Zeb2/Sip1 gene expression during Snail1-induced epithelial-mesenchymal transition. Genes Dev. 22, 756-769 (2008).

6. Ebralidze, A. K. et al. PU.1 expression is modulated by the balance of functional sense and antisense RNAs regulated by a shared cis-regulatory element. Genes Dev. 22, 2085-2092 (2008)

7. Hastings, M. L., Ingle, H. A., Lazar, M. A. \& Munroe, S. H. Post-transcriptional regulation of thyroid hormone receptor expression by cis-acting sequences and a naturally occurring antisense RNA. J. Biol. Chem. 275, 11507-11513 (2000).

8. Huarte, M. et al. A large intergenic noncoding RNA induced by p53 mediates global gene repression in the p53 response. Cell 142, 409-419 (2010).
9. Katayama, S. et al. Antisense transcription in the mammalian transcriptome. Science 309, 1564-1566 (2005).

10. Spigoni, G., Gedressi, C. \& Mallamaci, A. Regulation of Emx2 expression by antisense transcripts in murine cortico-cerebral precursors. PLOS ONE 5, e8658 (2010).

11. Setsuie, R. \& Wada, K. The functions of UCH-L1 and its relation to neurodegenerative diseases. Neurochem. Int. 51, 105-111 (2007).

12. Liu, Y., Fallon, L., Lashuel, H. A., Liu, Z. \& Lansbury, P. T. Jr. The UCH-L1 gene encodes two opposing enzymatic activities that affect $\alpha$-synuclein degradation and Parkinson's disease susceptibility. Cell 111, 209-218 (2002).

13. Barrachina, M. etal. Reduced ubiquitin C-terminal hydrolase-1 expression levels in dementia with Lewy bodies. Neurobiol. Dis. 22, 265-273 (2006).

14. Barrachina, M. et al. Amyloid- $\beta$ deposition in the cerebral cortex in dementia with Lewy bodies is accompanied by a relative increase in ABPP mRNA isoforms containing the Kunitz protease inhibitor. Neurochem. Int. 46, 253-260 (2005).

15. Choi, J. et al. Oxidative modifications and down-regulation of ubiquitin carboxylterminal hydrolase L1 associated with idiopathic Parkinson's and Alzheimer's diseases. J. Biol. Chem. 279, 13256-13264 (2004).

16. Nishihara, H., Smit, A. F. \& Okada, N. Functional noncoding sequences derived from SINEs in the mammalian genome. Genome Res. 16, 864-874 (2006).

17. Ponicsan, S. L., Kugel, J. F. \& Goodrich, J. A. Genomic gems: SINE RNAs regulate mRNA production. Curr. Opin. Genet. Dev. 20, 149-155 (2010).

18. Quentin, Y. Fusion of a free left Alu monomer and a free right Alu monomer at the origin of the Alu family in the primate genomes. Nucleic Acids Res. 20, 487-493 (1992).

19. Andrei, M. A. et al. A role for elF4E and elF4E-transporter in targeting mRNPs to mammalian processing bodies. RNA 11, 717-727 (2005).

20. Merrick, W. C. Eukaryotic protein synthesis: still a mystery. J. Biol. Chem. 285, 21197-21201 (2010).

21. Prasanth, K. V. et al. Regulating gene expression through RNA nuclear retention. Cell 123, 249-263 (2005).

22. Holcik, M. \& Sonenberg, N. Translational control in stress and apoptosis. Nature Rev. Mol. Cell Biol. 6, 318-327 (2005).

23. Gilbert, W. V. Alternative ways to think about cellular internal ribosome entry. J. Biol. Chem. 285, 29033-29038 (2010)

24. Yoon, A. et al. Impaired control of IRES-mediated translation in X-linked dyskeratosis congenita. Science 312, 902-906 (2006).

25. Malagelada, C., Jin, Z. H., Jackson-Lewis, V., Przedborski, S. \& Greene, L. A. Rapamycin protects against neuron death in in vitro and in vivo models of Parkinson's disease. J. Neurosci. 30, 1166-1175 (2010).

26. Santini, E., Heiman, M., Greengard, P., Valjent, E. \& Fisone, G. Inhibition of mTOR signaling in Parkinson's disease prevents L-DOPA-induced dyskinesia. Sci. Signal. 2, ra36 (2009)

Supplementary Information is available in the online version of the paper.

Acknowledgements We thank S.G. laboratory members for thought-provoking discussions and C. Leonesi for technical help. We thank F. Persichetti, A. Mallamaci, E. Calautti, S. Saoncella, A. Lunardi, D. De Pietri Tonelli, R. Sanges, M. E. MacDonald and T. Perlmann for support and discussions; and M. J. Zigmond and B. Joseph for sharing the MN9D cell line. This work was supported by the FP7 Dopaminet to S.G., E.S. and P.C., by The Giovanni Armenise-Harvard Foundation to S.G. and by the Compagnia di San Paolo to S.B.

Author Contributions C.C. designed and performed the experiments, and analysed the results; L.Ci. designed and performed the experiments, and analysed the results; M.B. designed and performed the experiments, and analysed the results; A.B. prepared polysomes; S.Z. designed the experiments, analysed the results and wrote the manuscript; S.F. carried out qRT-PCR on polysome fractions and the pulse labelling experiment; E.P. prepared polysomes and carried out northern blotting; I.F. analysed the results; L.Co. designed the experiments and analysed the results; C.S. analysed the data and discussed the results; A.R.R.F. performed bioinformatic analysis for the identification of SINEB2 and family members and designed $\triangle$ Alu and $\triangle$ SINEB2 mutants; P.C. provided reagents, experimental design and managing; S.B. designed polysome experiments, analysed the data and wrote the manuscript; E.S. performed bioinformatic analysis for the identification of S-AS pairs, designed experiments for the analysis of antisense Uchl1 expression and analysed the results; S.G. designed the experiments, analysed the results and wrote the paper.

Author Information Reprints and permissions information is available at www.nature.com/reprints. The authors declare competing financial interests: details are available in the online version of the paper. Readers are welcome to comment on the online version of the paper. Correspondence and requests for materials should be addressed to S.G. (gustinci@sissa.it). 


\section{METHODS}

Oligonucleotides. The complete list of oligonucleotides used for cloning and for quantitative real-time PCR experiments is included in Supplementary Information (Supplementary Fig. 12).

Plasmids. Full-length DNA sequence of antisense Uchl1 was amplified via fusion PCR starting from RACE fragment and FANTOM clone 6430596G22 (GenBank AK078321.1) with forward mouse antisense $U c h l 1 \mathrm{FL}$ and reverse mouse antisense Uchl1 FL primers.

Mouse Uchl1 mRNA was subcloned from FANTOM clone 2900059022 (GenBank AK013729.1) in the unique PmeI site of pcDNA3.1.

cDNA sequence of human antisense UCHL1 was amplified from a sample of human brain total RNA (Clontech, 636530) with the primers human $5^{\prime} \mathrm{F}$ and human $3^{\prime} \mathrm{R}$.

Oligonucleotides that target the sequence $-14 /+3$ around the TSS of antisense Uchll were annealed and cloned into pSUPERIOR.Neo.GFP vector (OligoEngine) in the BglII/XhoI site. Scrambled sequence was also cloned and used as control.

The antisense Uchl1 $5^{\prime}$ deletion mutant $\left(\Delta 5^{\prime}\right)$ was generated by PCR using the oligonucleotides forward mouse antisense $U \operatorname{chll}\left(\Delta 5^{\prime}\right)$ and reverse mouse antisense Uchl1 FL. PCR fragment was cloned in the unique EcoRI site in pcDNA3.1.

The antisense Uchl1 $3^{\prime}$ deletion mutant $\left(\Delta 3^{\prime}\right)$ was generated by PCR using the forward mouse antisense Uchll FL and reverse mouse antisense $U \operatorname{chll}\left(\Delta 3^{\prime}\right)$ primers and cloned in the unique EcoRI site in pcDNA3.1.

The antisense $U$ chll $(\triangle \mathrm{AS})(\Delta \mathrm{Alu}+$ SINEB2) mutant was obtained by subsequent cloning of PCR fragment I (NheI-EcoRI site) and PCR fragment II (EcoRI-HindIII site) into pcDNA3.1. Primers forward mouse antisense Uchl1 FL Nhe and reverse pre-SINE B2 EcoRI were used to generate fragment I; primers forward post-ALU EcoRI and reverse mouse antisense FL HindIII were used for PCR fragment II.

The antisense $U \operatorname{chl1}(\Delta \mathrm{A})(\Delta \mathrm{Alu}, 1000-1045)$ mutant was generated with a similar strategy to antisense Uchll( $\Delta \mathrm{AS})$. Forward mouse antisense Uchll FL NheI and reverse pre-ALU EcoRI were used for PCR fragment I; forward postALU and reverse mouse antisense FL HindIII for PCR fragment II.

The antisense $U \operatorname{chll}(\Delta S)(\Delta$ SINEB2, 764-934) mutant was obtained with a similar strategy to antisense $U$ chll $(\triangle \mathrm{AS})$. Oligonucleotides forward mouse AS Uchl1 FL NheI and reverse pre-SINE B2 EcoRI for fragment I; forward postSINE B2 EcoRI and reverse mouse AS FL HindIII for fragment II.

For antisense Uchll(ASf) (Alu + SINEB2 flipped), PCR fragment obtained with the primers forward SINEB2 inside and reverse Alu flip was cloned in the unique EcoRI site of antisense $U$ chll( $\triangle \mathrm{AS})$.

For antisense Uchl1(Sf) (SINEB2 flipped), PCR fragment obtained with forward SINE B2 inside and reverse SINE flip oligonucleotides was cloned in the unique EcoRI site of antisense Uchl1 $\triangle$ SINEB2.

For antisense Uchl1(73 bp), the method of 'annealing and primer extension' of two 3 '-end overlapping oligonucleotides was used to generate the 73-bp antisense Uchl1 overlap region. Annealed fragment was obtained with antisense Uchl1 $73 \mathrm{bp}$ forward and antisense Uchl1 73 bp reverse. Fragment was digested with XhoI and EcoRV and ligated into antisense Uchl1 $\Delta 5^{\prime}$ plasmid.

The antisense SCR 73-bp mutant was obtained with a similar strategy as antisense $U \operatorname{chll}(73 \mathrm{bp})$. The annealing extension was performed with oligonucleotides with scramble (SCR) sequence (antisense SCR forward and antisense SCR reverse).

Full-length mouse antisense $U x t$ was amplified by PCR starting from FANTOM clone 4833404H03 (GenBank AK029359.1) with specific primers (forward mouse antisense $U x t$ and reverse mouse antisense $U x t$ ). PCR fragment was subcloned into pcDNA3.1 using XbaI and HindIII restriction enzymes.

The antisense GFP plasmid was generated with a similar strategy as antisense Uchll(73 bp). Seventy-two base pairs corresponding to nucleotide $-40 /+32$ with respect to the ATG of GFP sequence in pEGFP-C2 vector (Clontech) were chosen as target sequence for artificial antisense DNA generation. For annealing, the GFP antisense forward and GFP antisense reverse primers were used.

Cells. MN9D cells were obtained from M. J. Zigmond. Cells were seeded in 100-mm dishes in Dulbecco's modified Eagle's (DMEM) medium containing 10\% fetal bovine serum (Invitrogen) supplemented with penicillin $\left(50\right.$ units $\left.\mathrm{ml}^{-1}\right)$ and streptomycin (50 units $\left.\mathrm{ml}^{-1}\right)$. For experiments, cells were plated in poly-L-lysine (P2636 Sigma) coated dishes and grown overnight. Approximately $50 \%$ confluent cells were treated with $1 \mu \mathrm{M}$ rapamycin (R0395, Sigma) or DMSO vehicle for $45 \mathrm{~min}$.

For the establishment of stable cell lines (shRNA $-15 /+4$, shRNA scrambled, pcDNA 3.1- and AS Uchl1 $\triangle$ SINEB2), MN9D cells were seeded in 100-mm Petri dishes and transfected with Lipofectamine 2000 (Invitrogen) according to the manufacturer's instruction. Stable clones were selected by $500 \mu \mathrm{M}$ neomycin (N1142, Sigma). HEK cells (Sigma) were cultured under standard condition in DMEM containing $10 \%$ fetal bovine serum supplemented with antibiotics. Transient transfections were done with Lipofectamine 2000 (Invitrogen). For al experiments, $\mathrm{S}$ and AS plasmids were transfected at 1:6 ratio.
Animal handling. All animal experiments were performed in accordance with European guidelines for animal care and following SISSA Ethical Committee permissions. Mice were housed and bred in SISSA/CBM non-SPF animal facility, with $12 \mathrm{~h}$ dark/light cycles and controlled temperature and humidity. Mice had ad libitum access to food and water. C57BL/6 male mice $(n=5), 8-10$ weeks old, were used for in situ hybridization experiments. Laser capture microdissection (LCM) of dopaminergic neurons was performed on 8-10-week-old male TH-GFP/21-31 mice $(n=3)$. Intra-cardiac perfusions were done under total anaesthesia.

RACE and multiplex RT-PCR. The 5' UTR of antisense Uchl1 was amplified by RACE PCR (GeneRacer, Invitrogen) by MN9D total RNA and cloned into pGEM-T Easy vector (Promega).

qRT-PCR. Total RNA was extracted from cells and mouse tissue samples (adrenal gland, cerebellum, cortex, olfactory bulb, striatum, ventral midbrain, heart, colon, kidney, lung, lymph node, ovary, prostate, spleen, testis, uterus) using Trizo reagent (Invitrogen) according to the manufacturer's instruction. An RNA panel of 20 different normal human tissues (pools consist of at least three tissue donor with full documentation on age, sex, race, cause of death) was obtained from Ambion (AM6000). All RNA samples were subjected to DNase I treatment (Ambion). A total of $1 \mu \mathrm{g}$ of RNA was subjected to retrotranscription using iScript cDNA synthesis kit (BioRad) and Real Time qRT-PCR was carried out using SYBR green fluorescence dye ( $2 \times$ iQ5 SYBR Green supermix, BioRad) TATA-binding protein (TBP) and RNA polymerase II (RPII) were used as housekeeping genes to normalize different mouse and human tissues as tested by the GeNorm program, version 3.5 (http://medgen.ugent.be/genorm/) ${ }^{27}$. GAPDH and $\beta$-actin were used as normalizing controls in all the other qRT-PCR experiments. The amplified transcripts were quantified using the comparative Ct method and the differences in gene expression were presented as normalized fold expression $(\Delta \Delta \mathrm{Ct})$. All of the experiments were performed in duplicate. A heat map graphical representation of rescaled normalized fold expression $\left(\Delta \Delta \mathrm{Ct} / \Delta \Delta \mathrm{Ct}_{\max }\right)$ was obtained by using Matrix2png (http://www.bioinformatics.ubc.ca/matrix2png/). A list of oligonucleotides used for qRT-PCR experiments is in Supplementary Fig. 12.

LCM technology. For LCM, regions of midbrain from TH-GFP/21-31 mice were dissected and incubated in $1 \times$ Zincfix solution for $6 \mathrm{~h}$. They were then cryoprotected in $30 \%$ sucrose solution at $4{ }^{\circ} \mathrm{C}$ overnight, embedded in Neg-50 section medium, snap-frozen and left to equilibrate in a cryostat chamber at $-21^{\circ} \mathrm{C}$ for $1 \mathrm{~h}$ before sectioning, as described earlier ${ }^{28}$. Cryostat $14 \mu \mathrm{m}$ midbrain coronal sections were thaw-mounted on Superfrost plus glass slides (Mezzle-Glasser) and dopaminergic $\mathrm{GFP}^{+}$cells were gathered via LCM and collected in microfuge (PALM adhesive caps). RNA was immediately extracted using the Absolutely RNA Nanoprep kit (Stratagene), eluted in RNase/DNase free water (Ambion) and retro-transcribed.

Two-colour in situ hybridization. After perfusion with $4 \%$ formaldehyde, mouse brain was cryoprotected overnight in 30\% sucrose. In situ hybridization was performed on cryostat slices $(16 \mu \mathrm{m})$. Sense and antisense probes were generated by in vitro transcription from the cDNA encoding the distal $600 \mathrm{bp}$ of mouse Uchl cDNA and the last 1,000 bp of mouse antisense Uchl1. The probes for Uchll and antisense Uchl1 were labelled with digoxigenin (DIG labelling, Roche) and biotin (BIO-labelling mix, Roche), respectively. Incorporation of biotin and digoxigenin was checked via a northern blot assay. In situ hybridization was performed as described previously ${ }^{29}$. Slices were pre-treated with $3 \%$ hydrogen peroxide for $30 \mathrm{~min}$. Hybridization was performed with probes at a concentration of $1 \mu \mathrm{g} \mathrm{ml}^{-1}$ (Uchll) and $3 \mu \mathrm{g} \mathrm{ml}^{-1}$ (antisense Uchl1) at $60^{\circ} \mathrm{C}$ for $16 \mathrm{~h}$. For biotinylated RNA detection, streptavidin-HRP (Amersham Bioscience) was used (1:250) for $2 \mathrm{~h}$ in TNB buffer (Tris $\mathrm{HCl} \mathrm{pH} 7.5100 \mathrm{mM}, \mathrm{NaCl} 150 \mathrm{mM}, 0.5 \%$ blocking reagent), and signals were visualized using the TSA Cy3 system (Perkin Elmer) after washing in TNT buffer (Tris $\mathrm{HCl}$ pH $7.5100 \mathrm{mM}, \mathrm{NaCl} 150 \mathrm{mM}, 0.05 \%$ Tween-20). In situ hybridization on DIG-labelled probe was performed with monoclonal anti-DIG antibody after TSA reaction. To combine RNA in situ hybridization with immunofluorescence, slices were incubated with anti-tyrosine hydroxylase (TH) antibody 1:1,000 (Chemicon). Signals were then detected with fluorescent dye-conjugated secondary antibody goat anti-rabbit 405 and goat anti-mouse 488 . Sections were then washed, mounted with Vectashield (Vector lab) mounting medium and observed with a confocal microscope (Leica).

Western blot. Cells were lysed in SDS sample buffer $2 \times$. Proteins were separated in $15 \%$ SDS- polyacrylamide gel and transferred to nitrocellulose membranes. Immunoblotting was performed with the following primary antibodies: antiUCHL1 (3524 Cell Signaling), anti-UXT (11047-1-AP Proteintech Group), antip53 (1C12) monoclonal antibody (2524, Cell Signaling) and anti- $\beta$-actin (A5441, Sigma). For the mTOR pathway: anti-phospho-p70 S6 kinase (Thr 389) (9234), anti-phospho-4E-BP1 (Ser65) (9451), anti-p70 S6 kinase (9202), anti-4E-BP1 (9452), anti-phospho-Akt (Ser 473) (3787) were all purchased from Cell Signaling. Signals were revealed after incubation with recommended secondary antibodies 
conjugated with horseradish peroxidase by using enhanced chemiluminescence for UCHL1 (WBKLS0500 Immobilion Western Chemioluminescent HRP substrate) and ECL detection reagent (RPN2105, GE Healthcare).

Protein stability. MN9D cells were seeded in 12-well plates overnight and then exposed to $100 \mu \mathrm{g} \mathrm{ml}^{-1}$ protein synthesis inhibitor cycloheximide (CHX) for $15 \mathrm{~min}$ and rapamycin $1 \mu \mathrm{M}$ or DMSO vehicle control for the following $45 \mathrm{~min}$. Cellular fractionation. Nucleo-cytoplasmic fractionation was performed using Nucleo-Cytoplasmic separation kit (Norgen) according to the manufacturer's instruction. RNA was eluted and treated with DNase I. The purity of the cytoplasmic fraction was confirmed by qRT-PCR on pre-ribosomal RNA.

Polysome profiles. Polysome profiles were obtained using sucrose density gradients. MN9D cells were treated with $1 \mu \mathrm{g} \mathrm{ml}^{-1}$ rapamycin for $35 \mathrm{~min}$, then with $100 \mu \mathrm{g} \mathrm{ml}^{-1}$ cycloheximide for $10 \mathrm{~min}$ prior to lysis in $150 \mu \mathrm{l}$ lysis buffer $(50 \mathrm{mM}$ Tris- $\mathrm{HCl} \mathrm{pH} \mathrm{7.5,} 100 \mathrm{mM} \mathrm{NaCl}, 30 \mathrm{mM} \mathrm{MgCl}_{2}, 100 \mu \mathrm{g} \mathrm{ml}^{-1}$ cycloheximide, $0.1 \%$ NP-40, $40 \mathrm{U} \mathrm{ml}^{-1} \mathrm{RNasin}$, protease inhibitors cocktail). Whole-cell extracts were clarified at $4{ }^{\circ} \mathrm{C}$ for $10 \mathrm{~min}$ at $15,000 \mathrm{~g}$. The equivalent of $5-10$ absorbance units at $254 \mathrm{~nm}$ of the clarified cell extract was layered onto $15-55 \%(\mathrm{w} / \mathrm{v})$ sucrose gradient (50 mM Tris/acetate $\mathrm{pH} 7.5,50 \mathrm{mM} \mathrm{NH}_{4} \mathrm{Cl}, 12 \mathrm{mM} \mathrm{MgCl}_{2}$ and $1 \mathrm{mM} \mathrm{DTT}$ ) and centrifuged for $3 \mathrm{~h} 30 \mathrm{~min}$ at 39,000 r.p.m. in a Beckman SW41Ti rotor at $4{ }^{\circ} \mathrm{C}$. The gradient was pumped out by upward displacement and absorbance at $254 \mathrm{~nm}$ was monitored using BioLogic LP software (Bio-Rad). One-millilitre fractions were collected, $1 \mathrm{ml}$ Trizol reagent (Invitrogen) was added and RNA was extracted following the manufacturer's instructions. A fixed volume of each RNA sample was then retro-transcribed and the percentage of mRNA in each fraction was calculated as relative $\mathrm{Ct}$ value to total RNA.

Metabolic labelling. MN9D were used for analysis of translational rate. Cells were seeded at sub-confluency in 6-well plates, and rapamycin $(1 \mu \mathrm{M})$ or DMSO stimulations were performed for $45 \mathrm{~min}$. Cells were labelled with $5 \mu \mathrm{Ci} \mathrm{ml}^{-1}$ of $\left[{ }^{35} \mathrm{~S}\right]$ methionine (Amersham Pharmacia Biotech) for $45 \mathrm{~min}$. Cells were lysed in standard lysis buffer (Tris- $\mathrm{HCl} 20 \mathrm{mM}, \mathrm{NaCl} 20 \mathrm{mM}, 0.5 \%$ Triton X-100) and centrifuged. Supernatants were trichloroacetic-acid-precipitated and filtered on glass fibre discs under vacuum. Discs were counted with scintillation fluid in a $\beta$-counter. Total proteins were measured with the standard BCA method. Rate of incorporation was expressed as $\mathrm{CPM} /$ total protein ratio (mean \pm s.d.). Experiments were done in triplicate.

Pulse labelling and immunoprecipitation. To monitor de novo protein synthesis, HEK cells were transiently transfected with pEGFP plasmid (Clontech) in combination with antisense GFP or empty control vector. After $24 \mathrm{~h}$, medium was replaced with methionine/cysteine-free DMEM for $1 \mathrm{~h}$. Then, cells were labelled with $100 \mu \mathrm{Ci} \mathrm{ml}^{-1}$ of $\left[{ }^{35} \mathrm{~S}\right]$ methionine/cysteine (EasyTag, Perkin-Elmer) for $1 \mathrm{~h}$. Labelled cells were collected, lysed in RIPA buffer $(150 \mathrm{mM} \mathrm{NaCl}, 50 \mathrm{mM}$ Tris pH 8, 1 mM EDTA, 1\% NP40, 0.5\% deoxycholic acid and 0.1\% SDS) and used for immunoprecipitation with anti-GFP antibody (Invitrogen) overnight. Immune complexes were isolated with protein G-sepharose beads (Amersham) and separated on $10 \%$ SDS-PAGE. Newly translated GFP was visualized by autoradiography. Densitometric analysis was performed on high-resolution images with Photoshop-CS5. Normalization was obtained relative to input.
Northern blot. Polysome fractions were digested with $100 \mu \mathrm{g} \mathrm{ml}^{-1}$ proteinase K in $1 \% \mathrm{SDS}$ and $10 \mu \mathrm{g}$ glycogen (Invitrogen) at $37^{\circ} \mathrm{C}$ for $1 \mathrm{~h}$. RNA was obtained by phenol/chloroform extraction and re-suspended in formaldehyde/formamide MOPS buffer. Samples were incubated for $5 \mathrm{~min}$ at $65^{\circ} \mathrm{C}$ before being loaded into formaldehyde $1 \%$ agarose gel and run at $90 \mathrm{~V}$ for $4 \mathrm{~h}$ at $4{ }^{\circ} \mathrm{C}$. RNA was transferred onto Amersham Hybond-XL nylon membranes and UV-crosslinked. A radiolabelled Uchl1-specific complementary RNA probe was transcribed from the same plasmid used for in situ hybridization, performing the reaction in the presence of $50 \mu \mathrm{Ci}$ of $\alpha-{ }^{32} \mathrm{P}$-UTP (Perkin-Elmer). After treatment with DNase I (Ambion), the labelled riboprobe was purified on RNeasy columns (Qiagen). Membranes were pre-hybridized for $3 \mathrm{~h}$ at $65^{\circ} \mathrm{C}$ with NorthernMax prehybridization/hybridization buffer (Ambion) supplemented with $50 \mu \mathrm{g} \mathrm{ml}^{-1}$ salmon sperm DNA (Invitrogen), and hybridized with UCHL1 riboprobe overnight at $65^{\circ} \mathrm{C}$ in the same buffer. After extensive washes (most stringent conditions were $0.2 \times \mathrm{SSC} / 0.1 \%$ SDS at $65^{\circ} \mathrm{C}$ ), membranes were exposed to autoradiography at $-80^{\circ} \mathrm{C}$ with intensifying screens. Bioinformatic analysis. For the identification of additional translational activator candidates, we searched for FANTOM3 full-length cDNAs that were non-coding RNAs and overlap the $5^{\prime}$ end of coding transcripts in a head-to-head configuration. The filtered set of 8,535 FANTOM3 ncRNA transcripts described previously ${ }^{30}$ was used as our starting point. Genomic locations of these ncRNA transcripts and RefSeq ${ }^{31}$ coding transcripts were extracted from the alignments in the UCSC Genome browser ${ }^{32}$ to identify a set of 788 coding-sense-non-coding-antisense pairs. ncRNAs were then checked by RepeatMasker to identify SINEB2-related sequences (http://www.repeatmasker.org). This analysis reduced the number of pairs to 127 protein-coding transcripts with overlap at the $5^{\prime}$ end (60 with a sense strand version of the repeat, 53 with an antisense version and 14 with both sense and antisense versions).

Alignment of the SINEB2-related elements was then carried out using Clustalw (http://www.ebi.ac.uk/Tools/clustalw2/index.html). From this analysis the antisense overlapping transcripts with a repeat most similar to the one of antisense Uchl1 as well as in the same orientation were chosen for experimental testing (antisense Uxt)

Statistical analysis. Statistical analyses were performed with paired two-tailed Student's $t$-test. Results are mean $(n \geq 3) \pm$ standard deviation (s.d.).

27. Vandesompele, J. et al. Accurate normalization of real-time quantitative RT-PCR data by geometric averaging of multiple internal control genes. Genome Biol. 3, RESEARCH0034 (2002).

28. Biagioli, M. et al. Unexpected expression of $\alpha$ - and $\beta$-globin in mesencephalic dopaminergic neurons and glial cells. Proc. Natl Acad. Sci. USA 106, 15454-15459 (2009).

29. Ishii, T.,Omura, M. \& Mombaerts, P. Protocols for two- and three-color fluorescent RNA in situ hybridization of the main and accessory olfactory epithelia in mouse. J. Neurocytol. 33, 657-669 (2004).

30. Nordström, K. J. et al. Critical evaluation of the FANTOM3 non-coding RNA transcripts. Genomics 94, 169-176 (2009)

31. Maglott, D. R., Katz, K. S., Sicotte, H. \& Pruitt, K. D. NCBI's LocusLink and RefSeq. Nucleic Acids Res. 28, 126-128 (2000).

32. Kent, W. J. et al. The human genome browser at UCSC. Genome Res. 12, 996-1006 (2002). 